Vol. $7, n^{\circ} 2 \mid 2003$

Varia

\title{
Jean-Noël Luc (dir.), Gendarmerie, État et société au
} XIX ${ }^{\mathrm{e}}$ siècle

Paris, Publications de la Sorbonne, 2002, 510 pp., ISBN 2859444491

\section{Renée Zauberman}

\section{OpenEdition}

\section{Journals}

Édition électronique

URL : https://journals.openedition.org/chs/557

DOI : $10.4000 /$ chs. 557

ISSN : $1663-4837$

\section{Éditeur}

Librairie Droz

\section{Édition imprimée}

Date de publication : 1 décembre 2003

Pagination : 107-116

ISBN : 2-600-008990-3

ISSN : 1422-0857

\section{Référence électronique}

Renée Zauberman, « Jean-Noël Luc (dir.), Gendarmerie, État et société au XIX siècle », Crime, Histoire \&

Sociétés / Crime, History \& Societies [En ligne], Vol. 7, n² | 2003, mis en ligne le 23 février 2009,

consulté le 22 mars 2022. URL : http://journals.openedition.org/chs/557 ; DOI : https://doi.org/ 10.4000/chs.557

Ce document a été généré automatiquement le 22 mars 2022

(c) Droz 


\title{
Jean-Noël Luc (dir.), Gendarmerie, État et société au XIX ${ }^{\mathrm{e}}$ siècle
}

Paris, Publications de la Sorbonne, 2002, 510 pp., ISBN 2859444491

\author{
Renée Zauberman
}

\section{RÉFÉRENCE}

Jean-Noël Luc (dir.), Gendarmerie, État et société au XIXe siècle, Paris, Publications de la Sorbonne, 2002, 510 pp., ISBN 2859444491.

1 Les quelques cinq cents pages de Gendarmerie, État et société au XIX ${ }^{e}$ siècle, sous la direction de Jean-Noël Luc vont être doublement précieuses : pour commencer, elles closent enfin, nous semble-t-il, le long chapitre des pleurs que nous avons dû essuyer sur la faiblesse de la recherche sur la gendarmerie; ces lamentations étaient devenues un exercice obligé, auquel sacrifie d'ailleurs une dernière fois, espérons-le, le directeur de cette publication dans son introduction. Encore expose-t-il de façon circonstanciée les raisons du retard de ces chantiers : ce que l'on savait déjà de l'allergie des milieux intellectuels à l'égard de la violence d'État et des valeurs militaires (p.16) et de la place étouffante qu'occupait une " ego-histoire " gendarmique très apologétique, comme des résistances de l'institution aux regards venus d'ailleurs est utilement complété par ce que l'on devinait moins: le mépris pour le non-combattant des historiens du fait militaire qui prétendent étudier "tout ce qui prépare et concourt au combat », voire leur hostilité à l'égard de l'auxiliaire de la police et de la justice militaires. Toutes explications auxquelles il faut ajouter que le cloisonnement des disciplines universitaires en France n'a guère aidé les chercheurs à s'attaquer à cet objet d'un autre type parce qu'il broche sur le politique, le juridique, le militaire, le social... Exeunt, donc les lamentations.

Deuxième raison du prix que nous devons attacher à ce lourd ouvrage : s'éloignant d'un bon pas d'un passé de misère scientifique, appuyé sur la politique d'ouverture des fonds d'archives pratiquée par l'institution sans laquelle la curiosité de l'historien se trouve 
bien démunie, il pèse son poids de travaux tous azimuts, aussi plein de réponses que gros de questions qui laissent augurer d'un bel avenir pour ce chantier. Mieux encore, alors que le lecteur risquerait d'être débordé par la diversité et la richesse des vingtsept communications, Jean-Noël Luc le reçoit à l'entrée de chacune des cinq parties de l'ouvrage, pour l'introduire, avec un remarquable esprit de synthèse, aux questions traitées, en s'appuyant autant sur les travaux présentés que sur la recherche existant par ailleurs.

3 La première partie, Le bras armé de l'État, est introduite par ce que Jean-Noël Luc appelle un survol sélectif des textes organiques de la gendarmerie du $\mathrm{XIX}^{\mathrm{e}}$ siècle : contextes politiques de l'émergence de ce considérable corpus juridique, aperçus de la construction d'ordre au double visage, franchement militaire et progressivement judiciaire, questions sur les changements d'un corps qui n'a d'autre conception de luimême que celle d'une longue permanence historique, la réflexion est tirée au delà d'un incontournable exercice de cadrage juridique.

Des sept contributions de cette partie se dégage l'image de la vieille institution d'ordre toujours en train de se construire et toujours en concurrence avec d'autres. Un aspect particulier et tout à fait essentiel de cette construction est celle de la représentation que le corps a de lui-même, abordée par Marie-Berthe Servier dans un chapitre sur l'Historique de la Garde Républicaine. Cette mémoire officielle conçue à la Belle Époque bâtit, au détriment de toute vérité historique le mythe d'un corps continûment républicain et gendarmique. Si la garde doit ainsi "s'inventer ", c'est que constamment exhibée dans la liturgie de la République, elle doit assumer une charge symbolique qu'elle ne maitrise pas.

5 Jacques Lorgnier montre comment la Couronne a, depuis le $\mathrm{XVI}^{\mathrm{e}}$ siècle doté la Maréchaussée d'une organisation et de missions conservées, depuis, dans l'héritage presque génétique de la Gendarmerie nationale: une force militaire veillant à la sécurité publique et au respect des lois sur tout le territoire et y assurant une part importante de la police judiciaire (p.52). Par delà les secousses révolutionnaires, Bernard Gainot lit dans la loi organique de 1798, par sa référence appuyée et explicite à l'ordonnance de 1778, la perspective de consolidation de l'État moderne: certes, la République Constitutionnelle veut soumettre sa force publique au contrôle de la représentation nationale, mais elle la veut toujours mieux établie sur tout le territoire : la revendication par les population d'une brigade de proximité est en effet générale dès les cahiers de doléances, car dans une France encore essentiellement rurale, la police au village était un enjeu majeur. C'est donc au niveau local que Fabien Gaveau étudie, pour le début du $\mathrm{XIX}^{\mathrm{e}}$ siècle une première concurrence à laquelle est soumise la gendarmerie, celle des gardes champêtres : on voit se dessiner le conflit de fond entre pouvoir d'État et pouvoirs locaux: les gardes champêtres pratiquent de préférence l'arrangement amiable au sein d'une micro-société dont ils partagent la perception du quotidien, alors que les gendarmes représentent la loi impersonnelle de l'État qui tolère mal la survie de coutumes jugées archaïques.

6 Au sommet de l'État, c'est un autre conflit que met en scène Aurélien Lignereux entre Moncey, Inspecteur général de la gendarmerie et Fouché, ministre de la Police générale, qui montre bien le prix attaché à Paris à la distribution sur le territoire d'une 
force centralisée, tout lacunaire que fût encore son maillage dans les premières années du XIX ${ }^{e}$ siècle. Fouché ne pouvait guère prétendre assurer avec les seules forces du ministère de l'Intérieur la mission de surveillance politique. Le coup de force de sa directive de 1805 qui prétendait faire de la gendarmerie la sous-traitante de la police en matière de renseignement et son bras armé pour l'emploi de la force publique était l'hommage de la faiblesse à la force. Napoléon lui-même, soucieux d'équilibrer les pouvoirs, para cette attaque en mettant en avant son «affection toute paternelle pour ses gendarmes » (p.75) et la qualité de leurs informations, faits nus collectés avec zèle sur tout le territoire et à lui transmis avec célérité.

7 Soumettre plus complètement la gendarmerie à l'autorité civile, jusqu'à envisager sa démilitarisation, fut une passion française - on pourrait à vrai dire en parler au présent $\mathrm{du} \mathrm{XXI}^{\mathrm{e}}$ siècle - et Xavier Borda en détaille plusieurs épisodes au cours de la III ${ }^{\mathrm{e}}$ République: l'assaut "républicain" contre l'armée, suite à la défaite, fut assez aisément repoussé par le projet de Revanche; mais surtout, les assauts policiers furent récurrents contre un corps jugé inefficace dans ses missions de police parce que trop absorbé par ses tâches militaires. La gendarmerie répondait régulièrement à ces attaques en demandant un renforcement de ses moyens et en défendant son statut spécifique, faisant valoir que seule la discipline militaire pouvait faire tenir ensemble un service assuré par des milliers d'unités dispersées sur le territoire national. Reste que la crise sécuritaire du tournant des $\mathrm{XIX}^{\mathrm{e}}$-XXe siècles - affaire Vacher, bandes criminelles comme les chauffeurs de la Drôme - amena à dresser un piètre bilan de son action, qu'examine Jean-Marc Berlière. Le recrutement était médiocre en raison de la faiblesse des soldes et de l'absence de perspectives de carrière, sans compter le faible attrait d'un corps qui apparaissait débordé de travail, rigide et paperassier. La balance entre ses différentes missions continuait de pencher du côté des tâches militaires, au détriment des tâches de police judiciaire; de surcroît, le contrôle de l'ordre public qu'appelaient les manifestations, grèves, émeutes qui se multipliaient au début du siècle et qui dégarnissaient les unités de leurs hommes n'était sans doute pas sans conséquences sur la sécurité des campagnes. Si la gendarmerie se plaignait déjà d'être la bonne à tout faire de toutes les administrations, il était alors quelque chose qui semble la distinguer de la gendarmerie d'aujourd'hui, le désintérêt pour la chose policière : la notion de sécurité publique ne s'était pas encore rabattue sur la chasse au délinquant et l'abandon de l'uniforme qui apparaissait à d'aucuns comme le seul moyen d'y participer efficacement soulevait les résistances farouches d'une identité blessée. Le sentiment général étant cependant que la gendarmerie restait un instrument irremplaçable qu'il fallait se garder de toucher "sous peine de la déformer », un effet paradoxal de ces critiques fut que c'est l'institution policière qui, cette fois-là, récolta la mise avec la création, en 1908, des "brigades du Tigre", force mobile à large compétence territoriale spécialisée dans les enquêtes sur les crimes et délits. Il fallut attendre 1921 pour que la gendarmerie territoriale se vît déchargée du poids du maintien de l'ordre par la création des pelotons mobiles.

8 Les villes, les champs, les armées, voilà nos gendarmes sur les théâtres d'opération qui sont l'objet de la deuxième partie de l'ouvrage. Jean-Noël Luc l'introduit par un examen détaillé, appuyé en partie sur des séries statistiques longues, de l'évolution quantitative du corps, dont les agents se multiplièrent par six au cours de ce long XIX ${ }^{e}$ siècle, mais qui étaient déjà, à la fin de la monarchie de Juillet, quatre fois plus nombreux qu'à la veille de la Révolution et représentaient dès lors un des visages les plus visibles de l'État dans la société. Les exigences du développement économique et 
de la croissance démographiques conduisirent à l'évidence à l'étoffement du corps, mais y participèrent aussi des choix politiques comme ceux de la Seconde République conservatrice ou plus tard de l'Ordre Moral.

9 L'examen de la politique d'implantation des brigades fait ressortir un thème vedette de l'histoire et de la sociologie de la gendarmerie, le conflit entre les autorités centrales qui prétendent pour la création ici ou la suppression là de brigades, défendre "l'intérêt général de la sûreté publique » - et les pouvoirs locaux qui cherchent pour leur sécurité à s'attraire une ou à garder «leur» brigade. Sans surprise, la trame cantonale des implantations se doublait du canevas que dessine la surveillance des voies de communication, mais Jean-Noël Luc signale, à travers une recherche de Cyril Cartayrade non publiée dans le volume, un point d'ancrage souvent oublié, la ville: l'exemple de Lyon au cours de la première moitié du siècle est celui d'une municipalité où la gendarmerie pesait moins par sa mission de surveillance, sans parler même de police judiciaire, que par son rôle dans la gestion des manifestations publiques. Cavaliers, discipline militaire et indépendance à l'égard de la municipalité en faisaient des agents efficaces du maintien ou du rétablissement de l'ordre, si bien que l'hypothèse de l'auteur fait du cadre urbain le laboratoire d'une professionnalisation dans cette fonction qui trouva son aboutissement dans la création mentionnée plus haut de la gendarmerie mobile en 1921.

10 Revenons au classique de l'implantation rurale. Étudiant le réseau du Sud-Ouest, JeanPaul Jourdan montre qu'on pouvait tirer argument de toute activité rassemblant des populations de façon habituelle - foires, marchés, étapes pour le passage des troupes ou des détenus - ou extraordinaire - chantiers de chemins de fer... - pour solliciter un poste de gendarmerie. On voit se profiler derrière ces exigences une obsession de l'ordre que partageaient les notables et souvent les préfets. L'attitude de la population restait plus incertaine : l'hostilité n'était pas rare, au moins jusqu'à la moitié du siècle; pendant longtemps, le gendarme est resté un étranger, mais progressivement, à mesure que le siècle s'avance, on voit apparaître dans les brigades des gendarmes de souche locale, signe d'une acceptation croissante de l'institution et, au-delà, pour le Sud-Ouest, d'une progressive intégration de la périphérie pyrénéenne à la nation.

11 La gendarmerie instrument d'une telle politique d'intégration? Hubert Heyries pose la question à propos de l'annexion de la Savoie en 1860. L'organisation de la gendarmerie de Savoie fut l'une des premières mesures impériales incorporant le duché à la France. Pour ne pas donner l'impression d'une troupe d'occupation, l'idée était d'absorber, dans les brigades françaises les gendarmes Piémontais. Si le projet reposait sur une bonne évaluation des enjeux de l'annexion, sa mise en œuvre fut finalement limitée par le désir d'assurer d'abord l'ordre impérial qui amena à recruter avant tout un corps fidèle à l'Empereur : les gendarmes finirent par être de plus en plus mal perçus par la population, parce qu'ils restaient les serviteurs des autres fonctionnaires français nommés en Savoie, qui, eux, se voyaient en mission de civilisation en pays barbare.

Ces barrières entre gendarmerie et population qu'elle police sont encore abordées dans deux articles de cette partie, l'un sur la gendarmerie au cours de l'épisode espagnol de Napoléon $\mathrm{I}^{\mathrm{er}}$, l'autre dans la conquête et la colonisation algériennes jusqu'à la veille de la Première Guerre mondiale. La mission de la gendarmerie d'Espagne (Nicole Gotteri), commandée par le Général Bucquet, consistait à assurer les communications entre la France et les corps d'armée dans les régions très accidentées du Nord de la péninsule ibérique et à maintenir l'ordre. Ces impératifs amenèrent le commandement à la 
recherche systématique du renseignement, tâche que devaient normalement remplir l'ensemble des unités cantonnées sur place. Les difficultés étaient de deux ordres : la dégradation de la situation militaire entraînait des interruptions de plus en plus fréquentes de la transmission des informations en provenance des escadrons, d'autant que - et on arrive là au cœur de problème -, dès 1811, personne parmi les populations terrorisées par les insurgés ne voulait plus se charger de porter les courriers. Quant à obtenir des renseignements de la population, si cela fut possible pendant quelques temps dans les territoires contrôlés, auprès de ceux qui soutenaient la cause du roi Joseph, là encore, à partir de 1811, il devint impossible de recruter des espions.

13 Sur une échelle beaucoup plus vaste, et pour un ensemble de missions plus complexes, le problème se reposa en Algérie. André-Paul Comor montre la mutation réalisée entre la gendarmerie d'Afrique des opérations de guerre des premières décennies et la gendarmerie de la veille de la Première Guerre mondiale, sédentarisée et assurant une surveillance plus étroite des campagnes autour des villages européens et des douars indigènes. Au long du siècle, la gendarmerie connut de l'autre côté de la Méditerranée des mutations qui ressemblaient aux étapes de son évolution métropolitaine : contrôle d'un corps expéditionnaire turbulent, progressif tissage d'un réseau de brigades sur un territoire qui s'étendait et donc auprès d'une population qui se multipliait et qui apparaissait très clivée entre Européens et Algériens. La méthode gendarmique de base - connaître le pays, en dresser les cartes, se renseigner sur les habitants - se heurtait à l'obstacle de la situation coloniale: comment s'informer dans et sur une population dont on ignorait la langue et les coutumes? Le projet de création de brigades mixtes composées de gendarmes français et d'auxiliaires autochtones n'aboutit qu'à un corps d'auxiliaires de cavaliers algériens anciens militaires, qui répondait mal aux besoins d'une force civile. Au tournant du siècle, au moment où l'assimilation au modèle administratif métropolitain était pourtant acquise, la grande crise d'insécurité que connut l'Algérie mit en lumière les insuffisances et les faiblesses du dispositif destiné à assurer avant tout la sûreté des communications entre les centres de colonisation et par là même, la sécurité des Européens isolés : révoltes indigènes, délinquance de bandes mobiles bénéficiant du soutien de la population algérienne, les gendarmes furent mis à rude épreuve et durent affronter la critique violente - allant jusqu'à la demande de suppression du corps - de leur inefficacité dans la surveillance préventive et répressive des délits commis par les autochtones.

"Veiller à la sûreté publique et assurer le maintien de l'ordre et l'exécution des lois ", Jean-Noël Luc introduit dans la troisième partie de l'ouvrage ce vaste programme, repris de décret en loi organique qui oblige l'historien à regarder de près les multiples missions auxquelles la gendarmerie s'adonne, inscrivant son travail au long des jours, des années et finalement du siècle dans la " construction de l'État envisagé comme un long processus de centralisation administrative et d'encadrement de la société et de l'individu» (p. 198). Retenons deux questions essentielles ici considérées : comment mesurer, à travers les archives, ce qu'ont véritablement fait les gendarmes, dans la masse des tâches qui leur étaient enjointes par un État qui nourrissait en toute innocence des rêves de toute-connaissance, de toute-puissance? Deuxième question, peut-on évaluer les progrès de la construction de la France contemporaine à l'aune des relations qui se sont, au cours du siècle, nouées entre ces émissaires d'un pouvoir encore lointain qu'étaient les gendarmes et des sociétés rurales toujours ancrées dans des systèmes de pouvoirs locaux? 
Sept articles examinent ces questions sous différents angles: la surveillance des populations peut se présenter sous son aspect politique. Certes, le ministère de la Police Générale sous la Monarchie censitaire savait que l'usage des «mouchards " travaillant dans l'ombre pour de l'argent était sans pertinence pour un corps exerçant dans tout l'éclat de son uniforme. Mais justement, c'est en cela que cette surveillance de l'« esprit public » faisait figure auprès de la population de «bonne surveillance», franche, dévouée, désintéressée. Si l'on ajoute à cela que la distribution des brigades sur le territoire fournissait l'atout de la proximité, Pierre Karila-Cohen peut montrer que l'instrument gendarmique fut finalement efficace dans la collecte du renseignement politique. D'autant que le concept du "politique » se découpait encore assez mal, en cette première partie du siècle, sur le fond de la vie sociale telle qu'elle était « naturellement » observée par les brigades : des routes sûres, des crimes et délits peu nombreux, une mendicité faible, tels étaient les éléments essentiels de la tranquillité, d'un « esprit public » satisfaisants.

L'étude, pour la seconde partie du siècle, de l'efficacité du travail de prévention des troubles divers dans le monde rural aquitain montre que, prévention des mouvements sociaux, grèves, violences au cours de foires ou à la sortie du cabaret, le travail de fond de la gendarmerie était sensiblement le même: surveillance des individus "à risques »- agitateurs politiques, anarchistes, étrangers, braconniers, marchands ambulants, gens du voyage -, mais surveillance aussi des foires et marchés, des charivaris et autres manifestations populaires. Corinne Marache décrit des brigades qui, pour glaner les informations sur tous ces risques, passaient un temps infini à parcourir les campagnes, établissant des liens avec les populations en général et des personnages-clés du monde rural en particulier, comme les commerçants, les employés municipaux, le personnel des chemins de fer.

17 À vrai dire, les relations de la brigade de gendarmerie avec la population du territoire qu'elle poliçait s'organisaient autour de la profonde fracture entre sédentaires et errants. Trois articles montrent qu'aussi conflictuelle qu'elle pût être avec les premiers, elle se construisait néanmoins autour d'un certain modus vivendi. La mise hors circuit des malfaiteurs, le secours aux populations - tâche partagée avec les pompiers - étaient certes des terrains où il était facile de gagner les cœur des administrés. Mais à côtés de ces missions positives, la police de la chasse qui échoyait à la gendarmerie n'était pas la moindre des occasions de se faire des ennemis dans la société rurale des débuts de la monarchie de Juillet au coup d'État de Louis-Napoléon Bonaparte. De toutes parts, on réclamait la gendarmerie, contre les malfaiteurs, les déserteurs, pour empêcher les rixes, mais rarement pour réprimer le braconnage et les contrôles des permis de port d'arme ou de chasse peuvent aller jusqu'à l'échange de coups de feu. Ces violences ne doivent pas s'interpréter comme pur folklore dénié de sens politique: Christian Estève voit en tous cas une certaine concordance entre les départements qui se sont levés en nombre en décembre 1851 et ceux où la répression cynégétique était forte : il suggère qu'il y eut, dans les violences qui frappèrent alors les gendarmes au moins un mélange des genres qui aurait placé les braconniers du côté des démocrates par haine des forces de l'ordre.

Reste que l'ordinaire des interactions entre gendarmerie et population sédentaire ne conduisit pas toujours à ces extrémités. En trois « instantanés » fixés à partir de procèsverbaux de simple police trouvés dans les archives de la justice de paix du canton de Vitré, en 1835-1845, 1885 et enfin 1935, Jean-François Tanguy détaille les domaines de 
la vie quotidienne qu'étaient amenés à contrôler les gendarmes. Tout se passe comme si les pouvoirs publics, qui, en début de période visaient à contrôler la vie sociale dans toutes ses manifestations - routes, lieux de réunions, violences... - avaient progressivement renoncé à cette ambition. Si, dans le premier tiers du siècle, l'autorité des gendarmes était encore, et éventuellement violemment, mise en cause à propos de la chasse comme on l'a vu, ainsi que de la circulation routière, cinquante ans plus tard, il restait, au pire, des attitudes impertinentes ou peu amènes : le gendarme n'était plus l'ennemi et l'habitude de respecter les forces de l'ordre s'était répandue. D'ailleurs, la taille des patrouilles eut tendance à diminuer, leur sécurité n'étant plus vraiment en danger. En 1935, les mêmes conflits de la vie rurale avaient disparu des contraventions, l'écrasante majorité des interventions concernant les petites affaires de circulation.

19 Ce modus vivendi qui s'est négocié peu à peu, au long du siècle avec les populations sédentaires était bien éloigné de ce que Jean-François Wagniart décrit pour les vagabonds ou Henriette Asseo pour les Tsiganes. L'obsession de l'errance et de ses liens avec la criminalité tenait la III République, qui faisait peser sa demande de sécurité sur la gendarmerie chargée de la sûreté des campagnes et des voies de communication. Malgré les contradictions d'une politique qui hésitait, à la fin du siècle, entre répression et assistance, les errants restèrent les ennemis de ceux qui avaient été "placés dans l'espace public pour surveiller tout ce qui bouge ». Encore y avait-il une différence entre les vagabonds jetés sur les routes par les conditions du marché du travail et donc l'errance fluctuait avec celles-ci, et les Bohémiens et autres Tsiganes pour lesquels la politique de repérage et d'identification par le carnet anthropométrique eut un effet de renforcement de l'errance : en obligeant la famille à voyager ensemble, ce document a contribué à souder l'identité collective bohémienne, qui consiste à se mouvoir dans la société environnante, avec pour projet d'existence de faire des enfants tsiganes qui, à leur tour pourront se mouvoir avec aisance dans une société hostile et clairement identifiée comme telle.

La quatrième partie de l'ouvrage s'enfonce véritablement dans l'épaisseur sociale d'une institution construite par l'État pour être implantée au sein des sociétés locales. Le point d'interrogation dont Jean-Noël Luc assortit le titre «le gendarme, un soldat qui a pris racine ? » est là pour tenter de défaire le stéréotype du soldat-paysan, ou du moins, sur cette toile de fond, de dessiner des contrepoints comme le gendarme urbain. De travaux déjà classiques ou de ceux en train de se faire, il veut extraire des portraits riches des origines sociales des gendarmes, de leur taille comme de leur niveau d'instruction, de leur mariage, de leurs amitiés comme de leurs quelques biens, de leurs ambitions comme de celles de leurs enfants, tout cela inscrit dans les petites villes, les bourgs ou les villages où se sont déroulées leur carrières.

21 Les quatre articles de cette partie sont autant d'illustrations variées de ce programme. Des revues d'inspection menées en 1813 sur les sept compagnies de gendarmerie maritime, Pierre Lévêque tire un rapide portrait d'un corps un peu particulier: sans grands débouchés pour ses hommes en raison de son statut ambigu de gendarmerie placé sous l'autorité de la Marine, il rassemblait d'anciens soldats de la Royale originaires des départements côtiers; ces hommes ne constituaient probablement guère l'élite de l'arme, si l'on s'en tient à leur physique, à leur aptitude à l'écriture et à leur penchant pour la boisson.

22 Mesurées chez les 285 gendarmes de la compagnie du Nord pendant la première moitié $\mathrm{du}$ siècle, les trajectoires géographiques, professionnelles et sociales n'étaient pas 
foudroyantes. Devant ce que les contemporains décrivaient comme une crise sérieuse de l'arme, qui réservait à ses hommes un sort peu enviable - solde insuffisante, casernement insalubre, maigre retraite -, Arnaud-Dominique Houtte se demande quel fut l'intérêt des militaires qui choisirent cette affectation. Observant ce que l'on appellerait aujourd'hui le «turn-over » rapide des gendarmes entre les brigades, alors même que la mobilité verticale est faible, il conclut que la mobilité géographique s'inscrivait essentiellement dans une stratégie de retour au pays, le militaire ne s'engageant pas alors pour progresser dans l'échelle sociale. Horizon géographique et horizon social restent étroitement bornés et puisque le recrutement était très local, la sédentarité dans le pays natal valait sans doute promotion, d'autant que l'acquisition d'une pension de retraite à l'issue de la carrière faisait finalement de l'ex-gendarme un modeste rentier.

Si la mobilité était pour le gendarme pris individuellement une stratégie, elle pouvait poser à la gendarmerie un sérieux problème quant à l'implantation de l'institution au sein de la population. Un changement de focale permet aux auteurs des deux articles suivants d'examiner de près, en laissant de côté leur activité professionnelle, la place des gendarmes dans les sociétés locales de divers types : le canton de Tauves (Puy-deDôme), de la seconde Restauration jusqu'au début du I $^{\text {nd }}$ Empire d'une part, et la ville moyenne d'Alençon dans l'Orne tout au long du siècle, d'autre part.

$24 \mathrm{Si}$, pour Cyril Castayrade, l'ordre au village se construisait dans la dialectique entre distance et proximité, c'est cette dernière qu'il fait ressortir avant tout: le casernement, la rotation des hommes sont dans son analyse de moins de poids que leur enracinement. Du point de vue individuel, les alliances matrimoniales entre gendarmes et clans locaux satisfaisaient des intérêts réciproques : accès à une certaine aisance, voire à la notabilité grâce aux biens de l'épouse pour les premiers, accroissement de leur influence locale pour les seconds. D'un point de vue plus collectif, la présence de la brigade apportait une certaine prospérité au village: fournitures aux chevaux et entretien des bâtiments, consommation quotidienne permise par les revenus réguliers des gendarmes, protection des foires et marchés et de la sécurité des transactions qui s'y déroulaient. Tous ces avantages expliquent que la présence d'une brigade ait fini par devenir un enjeu dans la rivalité entre communes et que sous ces espèces particulières, l'intervention de l'État ait été de mieux en mieux acceptée. Reste que ces rapprochements, cette familiarité entre gendarmes et population a probablement joué davantage en faveur de ses strates sédentaires supérieures et que, pour en revenir au métier de gendarme, s'il y eut un certain amollissement de la fonction répressive, il fut corrélatif d'un durcissement de l'action contre le vagabondage.

Comparativement, à Alençon, le destin social des gendarmes paraît moins favorable, sur une période beaucoup plus longue, il est vrai : si au début du siècle, leur structure sociale était semblable à celle de la population de la ville, l'enrichissement industriel de la bourgeoisie locale finit par les laisser à la traîne. À partir du milieu du siècle, ils commencèrent à connaître une certaine forme d'aisance, mais guère plus que viagère et peu valorisante socialement: toujours casernés dans le vieux centre-ville qui se dégradait, ils représentaient encore une promotion sociale chez les ruraux comme pour les ouvrières qu'ils épousaient après les avoir rencontrées dans le quartier. Pour JeanLouis Lenhof, il s'agissait d'une "insertion sociale en demi-teinte »: issus de milieux modestes, les gendarmes ont échappé à la précarité sans parvenir à la notabilité qu'autorisait parfois le milieu rural. 
26 En six articles - deux sur la gendarmerie transalpine, un sur celle des Pays-Bas, deux sur celle d'Espagne et un dernier sur la gendarmerie belge, la cinquième et dernière partie de l'ouvrage présente un panorama de l'institution hors les frontières de la France, sinon de l'Empire. Question essentielle posée par Jean-Noël Luc dans son introduction: alors que bonne part de l'Europe s'était liguée contre l'entreprise impériale de Napoléon, pourquoi tant de pays y ont-ils repris à leur compte avec enthousiasme une de ses institutions les plus "coloniales", la gendarmerie? La réponse proposée est simplement fonctionnaliste : parce qu'ils en avaient besoin. Dans des territoires déstabilisés par les démobilisations des armées ou soumis au banditisme habituel aux pourtours de la Méditerranée, il fallait une force publique disséminée dans les campagnes; parce que les despotismes avaient besoin d'être défendus; parce qu'une institution hiérarchisée chargée de la police administrative et judiciaire contribuait, comme l'avait déjà écrit Emsley ${ }^{1}$, à montrer le pouvoir de l'État jusqu'au fond des campagnes les plus reculées, contre les systèmes de pouvoirs locaux. Bref, parce que, partout en Europe, quoique selon des rythmes différents, des puissances publiques centralisées se mettaient en place et que les gendarmeries en sont des instruments privilégiés.

Traitons à part l'article d'Axel Tixhon sur la mesure de la criminalité et l'essor de la gendarmerie belge entre 1841 et 1885 : il se distingue des autres par l'examen du travail de police judiciaire de cette dernière, mesuré à travers le volume des prévenus jugés pour délit. Il conclut que celui-ci était fonction tant de l'importance des effectifs que de la qualité du travail des brigades.

En dehors de ce point de vue particulier, les articles présentant l'exportation du modèle de la gendarmerie française font, à divers degrés une histoire politique et institutionnelle de l'implantation de l'arme: elle naquit directement des forces napoléoniennes au Piémont et aux Pays-Bas, pour s'indigéniser aussitôt après l'effondrement de l'Empire; elle se créa plus tardivement en Espagne, en s'inspirant explicitement de l'organisation française. En tout état de cause, toutes voulaient répondre au même souci: représenter un état central sur toute l'étendue de son territoire: Michael Boers décrit l'importance de la gendarmerie impériale, puis du Carabiniere Reale dans le rattachement à l'État piémontais, par la lutte contre le banditisme endémique qui y sévissait sous l'Ancien Régime, des périphéries méridionales mal contrôlées. La gendarmerie fut l'école de la nation : c'est à travers elle, estime Boers que les populations de ce pays, auparavant si turbulentes et mal gouvernées apprirent la présence d'un État fort et moderne. Même les Pays-Bas, que leur traditionnel attachement aux libertés locales rendaient pleins de détestation pour le centralisme napoléonien se reconnurent, dès 1814, immédiatement après le départ des Français, le besoin d'un corps destiné à contrôler troubles, échauffourées, soldats errants et criminels de tout poil et, plus généralement, à servir de bras armé pour l'exécution des ordres des autorités. Le bandolérisme qui avait développé son empreinte sur la vie quotidienne de l'Espagne de la première moitié du XIX siècle, l'instabilité politique et le problème carliste, tout cela mettait en cause la sécurité des routes et du commerce, voire l'autorité de l'État et ce sont bien ces problèmes qui servirent de justification aux projet de création de la Guardia Civil.

Toutes ces forces connurent au cours du siècle des difficultés semblables: elles se voulaient d'élite, et se heurtèrent aux problèmes de recrutement des hommes de troupe dans une population très peu alphabétisée, à des moyens financiers trop 
médiocres pour proposer des carrières attrayantes - la rotation des personnels fut un peu partout un frein à la professionnalisation-; la pauvreté de l'arme mit aussi longtemps hors d'atteinte l'objectif d'une installation à demeure des brigades sur l'ensemble des territoires, dans des casernes qu'il fallait construire : Jonathan Dunnage le mentionne pour l'Italie du dernier quart de siècle, le colonel Paz pour l'Espagne qui, à la fin du siècle, était loin d'avoir imposé la présence de la Guardia Civil dans la majorité des municipes.

Si l'aboutissement, un peu partout en Europe, fut l'acceptation par les populations de ces porteurs de la loi de l'État, seuls les articles sur l'Italie entrent un peu dans l'histoire de cette insertion, qui ne fut pas un long fleuve tranquille. Conflits et soutien mutuel firent longtemps le tissu ambigu des rapports entre gendarmerie et population: si les Piémontais du début du siècle voyaient bien l'avantage d'avoir à disposition, nuit et jour, une force qui protégeât leurs propriétés traditionnellement menacées par le banditisme, ils n'appréciaient guère sa recherche des insoumis et déserteurs ou sa lutte contre diverses contrebandes, sans parler même du comportement douteux d'hommes de troupe encore mal dégrossis. Mais rapidement, la pacification des campagnes fut l'argument définitif en faveur d'une institution qui ne s'intéressait pas seulement à la sécurité de l'État, mais aussi à celle de ses administrés. Plus tard, après l'unification, d'autres conflits s'élevèrent, quand des carabiniers jeunes et inexpérimentés, la tête farcie des notions aristocratiques sur la valeur de leur arme, eurent à policer le Sud infesté de brigands, ou à la fin du siècle, lorsqu'ils furent impliqués dans la surveillance des socialistes. Par ailleurs, dans les contextes locaux de clientélisme qui étaient l'ordinaire des relations politiques locales, les carabiniers étaient amenés à défendre les intérêts des élites contre l'agitation sociale et l'image que le corps cherchait à donner de lui-même, défenseur du peuple plutôt qu'instrument de répression, était généralement prise cum grano salis. Reste que tout brutaux que pussent se montrer les carabiniers quand la répression de mouvements de révolte devenait inévitable, il arrivait à la presse socialiste de les louer pour leur soutien au peuple - aide alimentaire ou modération dans des opérations de maintien de l'ordre.

Les quelques considérations de conclusion que Jean-Noël Luc a demandées à des historiens et des militaires suivent, à travers les prismes particuliers des uns et des autres, les fils rouges qui, depuis le titre Gendarmerie, État et société courent à travers les contributions au colloque: histoire d'une institution d'ordre, incarnée dans une histoire sociale, celle de ses hommes et des relations qu'ils entretiennent avec les populations qu'ils policent, le tout inscrit de façon constitutive dans l'histoire des États européens. Montrer que dans ces histoires s'ancrent encore les questions que posent aux scientifiques du temps présent la gendarmerie d'aujourd'hui fait de ce corps ce que peuvent souhaiter de plus stimulant les hommes de l'art : un objet historique vivant. 


\section{NOTES}

1. Emsley C. 1999, Gendarmes and the State in Nineteenth-Century Europe, Oxford, Oxford University Press (voir notre compte rendu de 2001 The French Gendarmerie: crossing sociological and historical perspectives, Crimes, Histoire \& Sociétés / Crime, History \& Societies, 5, 2, 149-156).

\section{AUTEURS}

\section{RENÉE ZAUBERMAN}

CESDIP - CNRS, zauberman@cesdip.com 